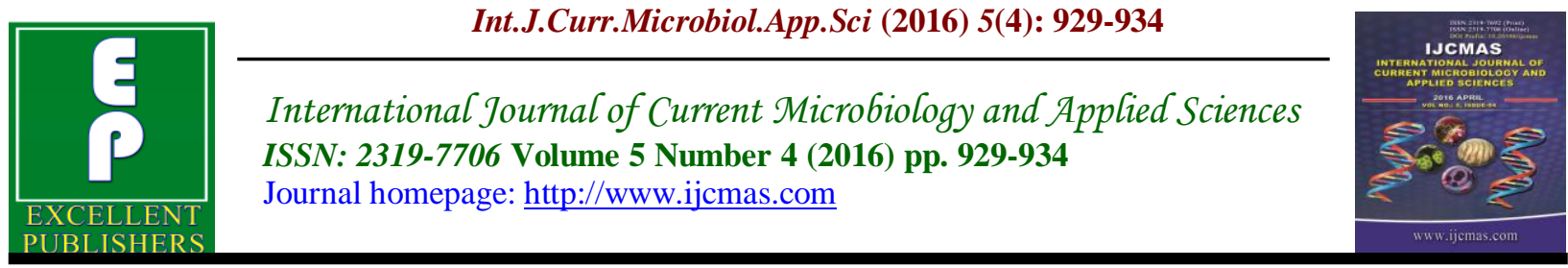

Original Research Article

http://dx.doi.org/10.20546/ijcmas.2016.504.105

\title{
Inducible Clindamycin Resistance among Clinical Isolates of Staphylococcus aureus in a Tertiary Care Centre, Kerala, India
}

\author{
I. R. Regha*, Deepa Harichandran and B. Sulekha \\ Department of Microbiology, Sree Narayana Institute of Medical Sciences, \\ Chalakka, Ernakulam, Kerala, India \\ *Corresponding author
}

\begin{abstract}
A B S T R A C T
Keywords

Clindamycin,

D test, MLSB

phenotype,

Staphylococcus

aureus.

Article Info

Accepted:

25 March 2016

Available Online:

10 April 2016

Drug resistance among Staphylococcus aureus is an increasing problem. Clindamycin is one of the effective antibiotic for treating both methicillin sensitive and resistant Staphylococcal infections. A major concern regarding the use of clindamycin therapy is the presence of inducible resistance. In vitro routine tests for clindamycin susceptibility may fail to detect inducible resistance resulting in treatment failure. The present study aimed to find out the inducible clindamycin resistance in the clinical isolates of Staphylococcus aureus using D test. The study was conducted at the Department of Microbiology in Sree Narayana Institute of Medical Sciences, Kerala over a period of one year. All of the total 220 S.aureus isolates during the period were included in the study. D test was done in the erythromycin resistant strains as per CLSI guidelines. Inducible clindamycin resistance was observed in $12.7 \%$, constitutive resistance in $8.1 \%$ and MS phenotype in $41.8 \%$ of the total isolates. The rate of MRSA and MSSA were $41.8 \%$ and $58.2 \%$ respectively. Inducible resistance and constitutive resistance were higer in MRSA (34.8\% and $10.9 \%$ respectively) as compared to MSSA where the inducible resistance was only $3.1 \%$ and no constitutive resistance was noticed. $19.6 \%$ of MRSA and 21.9\% of MSSA showed MS phenotype. Inducible clindamycin resistance was more common than constitutive resistance in our hospital. Both inducible and constitutive resistance showed higher incidence among MRSA than MSSA. The trends in resistance may vary from place to place. So Dtest should be done routinely to delineate different resistant phenotypes in the laboratory to help clinicians for the judicious use of clindamycin in order to avoid therapeutic failure as well as irrational use of higher antibiotics.
\end{abstract}

\section{Introduction}

Staphylococcus aureus is one of the most common cause of both community acquired and nosocomial infections. It includes minor cutaneous infections to life threatening conditions such as endocarditis, pneumonia and septicaemia (Lt.Col. Mahima Lall et al;2013, Naima Fasih et al; 2010). Emergence of methicillin resistant S.aureus (MRSA) which are resistant to $\beta$ - lactams as well as other classes of antibiotics often 
presents difficulty in treatment. The Macrolide- Lincosamide- Streptogramin B $\left(\mathrm{MLS}_{\mathrm{B}}\right)$ class of antibiotics is commonly used in the treatment of both MRSA and methicillin sensitive $S$. aureus (MSSA) infections (Prakash Sah et al ;2015). Among these drugs clindamycin is an attractive therapeutic option especially in skin and subcutaneous infections as they are available both as oral and intravenous preparations, have excellent tissue penetration, are relatively inexpensive and require no renal dose adjustments. It also inhibits the production of certain toxins and virulence factors by S.aureus . It is a useful choice in penicillin allergic patients. (Coyle EA et al ;2003, Prakash Sah et al ;2015). But the emergence of clindamycin resistance especially of inducible type due to the inappropriate use of $\mathrm{MLS}_{\mathrm{B}}$ antibiotic is becoming a major problem in its utility.

In Staphylococcus, Macrolide resistance arises either by ribosomal modification or efflux mechanism. The efflux pump is encoded by msr A gene which affects the macrolides and streptogramins and produces the MS phenotype.

The ribosomal modification is mediated by 23S r RNA methylase encoded by the erm genes. This methylase confers resistance to the $\mathrm{MLS}_{\mathrm{B}}$ class of antibiotics as the gene encodes methylation of the $23 \mathrm{~S} \mathrm{r} \mathrm{RNA}$ binding site that is shared by these drugs (Edward JE ;2010). Phenotypically such resistance may be constitutive $\left(\mathrm{cMLS}_{\mathrm{B}}\right)$ or inducible $\left(\mathrm{iMLS}_{\mathrm{B}}\right)$. In $\mathrm{cMLS}_{\mathrm{B}}$ phenotype methylase is always produced but in $\mathrm{iMLS}_{\mathrm{B}}$ phenotype it is produced only in the presence of an inducer, chiefly a macrolide. It is also possible for ribosomal mutations to occur spontaneously that transforms $\mathrm{iMLS}_{\mathrm{B}}$ strains to $\mathrm{cMLS}_{\mathrm{B}}$ phenotype without the presence of a macrolide inducer during clindamycin therapy (Leclercq $\mathrm{R}$ et al ;2002).
Constitutive resistant strains are easily identified by the routine antibiotic susceptibility test as they show resistance to both erythromycin and clindamycin. But the inducible clindamycin resistance is often missed unless D-test is done because the isolates show resistance to erythromycin but sensitive to clindamycin in the routine testing. Thus reporting $\mathrm{iMLS}_{\mathrm{B}}$ phenotypes as clindamycin sensitive by the microbiologist will mislead the clinician to start with clindamycin and treatment failure due to spontaneous mutations (Lewis JS et al; 2005). On the other hand a negative result of inducible clindamycin resistance helps the clinicians to consider clindamycin as a therapeutic option for erythromycin resistant strains (Nita Gangurde et al; 2014). A negative $\mathrm{D}$ test can also help the clinician to spare confidently the higher antibiotics like vancomycin and teicoplanin for treating non- life threatening MRSA infections. CLSI recommends $D$ test as a simple, reliable and inexpensive test to perform along with the routine susceptibility testing in order to detect inducible clindamycin resistance invitro ( CLSI ;2013, Gade N et al; 2013).

The prevalence of inducible clindamycin resistance varies by geographic location and bacterial species but reports are scanty from Kerala. The present study was undertaken to determine the percentage of inducible clindamycin resistance among S.aureus isolates in our locality by incorporating D test as a routine procedure in the clinical microbiology laboratory, in order to guide our clinicians for appropriate clindamycin therapy .

\section{Materials and Methods}

The study was conducted in a tertiary care centre, Kerala over a period of one year (March 2015- March 2016). All of the total 
220 Staphylococcus aureus isolates during the study period (from various clinical specimens like pus, wound swab, aspirates, blood and other sterile fluids) were included under the study. Antibiotic susceptibitity testing was done by Kirby Bauer's disc diffusion method on Mueller Hinton agar using Penicillin (10 U), Cefoxitin $(30 \mu \mathrm{g})$, Erythromycin $(15 \mu \mathrm{g})$, Clindamycin $(2 \mu \mathrm{g})$, Cotrimoxazole $(25 \mu \mathrm{g})$, Linezolid $(30 \mu \mathrm{g})$ and Vancomycin $(30 \mu \mathrm{g})$ as per CLSI guidelines (CLSI; 2013). Quality check for the disc were performed with ATCC Staphylococcus aureus 25923.

Isolates with cefoxitin zone size $\geq 22 \mathrm{~mm}$ were considerd as methicillin sensitive and those with zone size $\leq 21 \mathrm{~mm}$ were considered as methicillin resistant. The isolates that were found to be erythromycin resistant (zone $\leq 13 \mathrm{~mm}$ ) were further studied for inducible clindamycin resistance using 'D test" placing Erytromycin $(15 \mu \mathrm{g})$ and Clindamycin $(2 \mu \mathrm{g})$ disc at $15 \mathrm{~mm}$ apart. A flattening of the zone of inhibition adjacent to erythromycin discs after 18- 24 hours of incubation was considered as inducible clindamycin resistance (CLSI ;2013, Gade N et al; 2013).

Three different phenotypes were noted:

MS phenotypes : resistant to erythromycin (zone $\leq 13 \mathrm{~mm}$ ) and sensitive to clindamycin ( zone $\geq 21 \mathrm{~mm}$ )

iMLS $_{B}$ phenotype : erythromycin resistant (zone $\leq 13 \mathrm{~mm}$ ) and clindamycin sensitive (zone $\geq 21 \mathrm{~mm}$ ) showing D shaped zone around the clindamycin disc with flattening adjacent to erythromycin disc

cMLS $_{\mathbf{B}}$ phenotype : both erythromycin (zone $\leq 13 \mathrm{~mm}$ ) and clindamycin are resistant ( zone size $\leq 14 \mathrm{~mm}$ )

\section{Results and Discussion}

Out of the 220 S.aureus isolates ,138 isolates $(62.7 \%)$ were erythromycin resistant and 168 isolates ( $76.4 \%$ ) were clindamycin sensitive by routine antibiotic sensitivity testing. $54.5 \%$ of the total isolates were erythromycin resistant and clindamycin sensitive. By incorporating D test we got the the following phenotypes- MS phenotype in 92 isolates (41.8\%), c MLS $\mathrm{S}_{\mathrm{B}}$ in 18 isolates $(8.1 \%)$ and $\mathrm{i} \mathrm{MLS}_{\mathrm{B}}$ in 28 isolates ( $\left.12.7 \%\right)$. Methicillin resistance was noticed in 92 isolates (41.8\%) and 128 isolates ( 58.2\%) were MSSA. Among the MSSA, 32 isolates ( $25 \%$ ) were erythromycin resistant. Out of the total MSSA, MS phenotype was observed in 28 isolates (21.9\%), i $\mathrm{MLS}_{\mathrm{B}}$ phenotypes in 4 isolates $(3.1 \%)$ and no $\mathrm{cMLS}_{\mathrm{B}}$ phenotype was isolated. Sixty isolates $(65.2 \%)$ of MRSA were erythromycin resistant. MS phenotype was noticed in 18 isolates $(19.6 \%), \mathrm{c} \mathrm{MLS}_{\mathrm{B}}$ in 10 isolates $(10.9 \%)$ and $\mathrm{i} \mathrm{MLS}_{\mathrm{B}}$ in 32 isolates $(34.8 \%)$ out of the total MRSA .

The increasing frequency of Staphylococcal infections and changing pattern in antibiotic resistance have led to renewed interest in the use of clindamycin to treat such cases. Clindamycin is one of the effective agents for the treatment of skin and subcutaneous infections caused by MRSA and MSSA infections. (Lt.Col.Mahima Lall et al;2013, Naima Fasih et al ;2010). Inducible resistance to $\mathrm{MLS}_{\mathrm{B}}$ antibiotics especially to clindamycin needs special concern as spontaneous constitutively resistant mutants have been selected from such isolates both in vitro and in vivo during clindamycin therapy ( Venkata Raghavendra Rao et al; 2012, Kalpana Date et al ;2012).

In our hospital, with the routine sensitivity testing, clindamycin sensitivity is found to be $76.4 \%$ which reveals a chance of more 
clinical utility of clindamycin in our setting. $54.5 \%$ of this isolates were erythromycin resistant and clindamycin sensitive. But with $\mathrm{D}$ test, MS phenotype is only $41.8 \%$ of the isolates and the remaining $12.7 \%$ isolates is $\mathrm{iMLS}_{\mathrm{B}}$ phenotype.So fairly a big percentage would have been misinterpreted as clindamycin sensitive in the absence of the $\mathrm{D}$ test.Among the isolates, inducible clindamycin resistance is more common than constitutive resistance. The results of the present study is compared to some recent studies from India and other countries and is shown in Table:1. Even though there is slight difference in the percentages of resistance in different geographical areas majority of studies showed predominance of
$\mathrm{iMLS}_{\mathrm{B}}$ than c $\mathrm{MLS}_{\mathrm{B} .}$.

$41.8 \%$ of the total isolates were MRSA and $58.2 \%$ were MSSA. The prevalence of MRSA is different in various studies: Mehta et al; 2007 ,Vekata Raghavendra et al; 2012, Smita sood et al; 2013, Nita Gangurde et al; 2014, Prakash Sah et al; 2015, B Sasirekha et al; 2014, , shows percentage of isolation of MRSA as $26.6 \%, 75.27 \%, 7.75 \%, 32 \%$, $61.4 \%$ and $27.45 \%$ respectively.MRSA isolation is moderately high in our area. This difference in prevalence of MRSA among different countries and between different regions in a country may be due to varied population, geographical distribution and selection pressure in the community.

Table.1 Inducible and Constitutive Clindamycin Resistance in Various Studies

\begin{tabular}{|l|l|l|}
\hline & \multicolumn{2}{|c|}{ ( S. aureus $\mathrm{n}=220)$} \\
\hline & $\mathrm{i} \mathrm{MLS}_{\mathrm{B}} \%$ & $\mathrm{c} \mathrm{MLS}_{\mathrm{B}} \%$ \\
\hline Present study & 12.7 & $8.1 \%$ \\
\hline Gadepalli R et al $; 2006$ & 21 & 26.5 \\
\hline Smita sood $; 2013$ & 15.5 & 20.93 \\
\hline Nita Gangurde et al ;2014 & 13.53 & 12.61 \\
\hline Prakash et al; 2015 & 12.1 & 7 \\
\hline
\end{tabular}

Table.2 Inducible and Constitutive Clindamycin Resistance among MRSA and MSSA in Various Studies

\begin{tabular}{|l|l|l|l|l|}
\hline & \multicolumn{2}{|c|}{ MLS $_{\mathrm{B}}(\%)$} & \multicolumn{2}{c|}{ c MLS $_{\mathrm{B}}(\%)$} \\
\hline Present study & MRSA & MSSA & MRSA & MSSA \\
\hline $\begin{array}{l}\text { Venkata Raghavendra Rao et al; } \\
2012\end{array}$ & 45.71 & nil & 2.85 & nil \\
\hline Smita sood ;2013 & 62.5 & 60 & 38 & nil \\
\hline Nita Gangurde et al;2014 & 27.8 & 6.78 & 18.26 & 9.95 \\
\hline Prakash Sah et al ;2015 & 14 & 9.3 & 12.8 & nil \\
\hline
\end{tabular}


Table.3 MS Phenotypes in Various Studies

\begin{tabular}{|l|l|l|}
\hline & \multicolumn{2}{|c|}{ MS phenotypes } \\
\hline & MRSA (\%) & MSSA (\%) \\
\hline Present study & 19.6 & 21.9 \\
\hline Shantala et al $; 2012$ & 15.07 & 16.34 \\
\hline Smita sood $; 2013$ & 0 & 50 \\
\hline Nita Gangurde et al;2014 & 20.20 & 14.93 \\
\hline Prakash et al; 2015 & 19.8 & 14.8 \\
\hline
\end{tabular}

In our study both inducible and constitutive clindamycin resistance and MS phenotype were more in MRSA than MSSA. This is consistent with many studies from various regions. (Table:2 and Table:3). Almost all studies showed both inducible and constitutive resistance more in MRSA strains. In contrary, Schreckenberger et al and Levin et al reported higher incidence of inducible resistance in MSSA as compared to MRSA, $12.5 \%$ MRSA and 68\% MSSA respectively (Nita Gangurde et al 2014). $54.4 \%$ of total MRSA isolates were both erythromycin resistant and clindamycin sensitive by routine test. But by incorporating $\mathrm{D}$ test, Ms phenotype is only $19.6 \%$ and $34.8 \%$ are having inducible clindamycin resistance which can adversely affect the treatment with clindamycin. In MSSA also, $3.1 \%$ of isolates may be misinterpreted as sensitive and may lead to therapeutic failure.

So keeping in view of this relative high frequency of $\mathrm{iMLS}_{\mathrm{B}}$ phenotype especially among MRSA isolates, D test should be incorporated in the laboratory as a routine procedure. Providing a negative $\mathrm{D}$ test report from the lab will help clinicians to consider clindamycin for treating non life threatening Staphylococcal infections, especially for MRSA where there is only limited treatment options available. Thus use of higher antibiotics like vancomycin and linezolid can be reserved for complicated infections.
The incidence of resistance may be variable with geographical areas,study population and the hospital epidemiology. Hence there is definitely a need to monitor the local prevalenc of these resistance phenotypes by the microbiologists to guide the physicians in treating such cases judiciously and effectively.

\section{References}

Clinical and Laboratory Standard Institute;Performance standards for antimicrobial susceptibility testing, Wayne; 2013; $23^{\text {rd }}$ Informational Supplement: 33(1).

Coyle EA, Lewis RL, Prince RA. 2003.Iinfluence of clindamycin on the release of Staphylococcus aureus $\alpha$-hemolysis from methicillin resistant S. aureus: could MIC make a difference. Crit care Med.31 (suppl):A48

Edward JE. 2010. Macrolides, Clindamycin and Ketolides.In :Mandell GL, Bennett JE, Dolin R. (Ed). Mandell, Douglas and Bennetts Principles of practice of infectious diseases, seventh ed. Churchill Livingstone; $p$ p. 427-443

Gade N, Qazi MS. 2013. Inducible clindamycin resistance among Staphylococcus aureus isolates. Indian J of Basic \& Applied Med Res. 8(2) : 961-967

Gadepalli R, Dhawan B, Mohanty S, Kapil 
A, Das BK, Chaudhry R. 2006. Inducible clindamycin resistance in clinical isolates of Staphylococcus aureus. Indian J Med Res 123, April 2006: 571-573

Kalpana Date, Mamta Choudhary, Vilas Thombare. 2012. Inducible clindamycin resistance in clinical isolates of staphylococci in a rural hospital. Int J Biol Med Res. 3(3): 1922-1925

Leclercq R.2005. Mechanisms of resistance to macrolides and lincosamides: nature of the resistance elements and their clinical implications. Clin Infect Dis.34:482-92.

Lewis JS, Jorgensen JH. 2005.Inducible clindamycin resistance in staphylococci: should clinicians and microbiologists be concerned? Clin Infect Dis .40:280-5.

Lt Col Mahima Lall, Brig A. K. Sahni. 2013. Prevalence of inducible clindamycin resistance in Staphylococcus aureus isolated from clinical samples. Med J Armed Forces India :1-5

Mehta M, Dutta P, Gupta V. 2007. Bacterial isolates from burn wound infections and their antibiograms: an eight-year study. Indian J Plast Surg. 40 :25-28. Naima Fasih, Seema Irfan, Afia Zafar. 2010. Inducible clindamycin resistance due to expression of erm genes in Staphylococcus areus: Report from a tertiary care hospital, Karachi, Pakistan. JPMA. 60:750-53

Nita Gangurde, Preeti Bajaj, Sunita Phatale.
2014. Prevalence of Inducible clindamycin resistance among Staphylococcus aureus isolates in a tertiaryiary care hospital: an alarm "No antibiotic era". J of Evolution of Med and Dent Sci .3(18):4839- 4846

Prakash Sah, Rita Khanal, Pramila. 2015.Inducible and constitutive resistance in Staphylococcus aureus: an experience from Western Nepal. Int $\mathrm{j}$ of Bio med Res .6(05):316-319

Sasirekha, B., M. S. Usha, J. A. Amruta, S. Ankit, N. Brinda, R. Divya. 2014. Incidence of constitutive and inducible clindamycin resistance among hospital-associated Staphylococcus aureus.Biotech. 4 (1): $85-89$

Shantala GB, Shetty AS, Rao RK. 2011. Detection of inducible clindamycin resistance in clinical isolates of Staphylococcus aureus by the Disc Diffusion Induction Test. J Clin and Diagnost Res. 5 (1):35-37.

Smita Sood: 2013.Inducible clindamycin resistance among clinical Staphylococcal isolates from an Urban hospital in North West, India: Research and Review. J Med and Health Sci.2(4):64-67

Venkata Raghavendra Rao , A. Kavitha, K.S. Seetha. 2012.Prevalence of inducible clindamycin resistance among clinical isoslates of Staphylococci. National J Basic Med Sci. 2(1):68-71.

\section{How to cite this article:}

Regha, I. R., Deepa Harichandran and Sulekha, B. 2016. Inducible Clindamycin Resistance among Clinical Isolates of Staphylococcus aureus in a Tertiary Care Centre, Kerala, India. Int.J.Curr.Microbiol.App.Sci. 5(4): 929-934. doi: http://dx.doi.org/10.20546/ijcmas.2016.504.105 